\title{
Molecular authentication of multi-species honeysuckle tablets
}

\author{
C. Jiang*, Y. Yuan*, M. Chen and L. Huang \\ Institute of Chinese Materia Medica, Academy of Chinese Medical Sciences, \\ Beijing, China
}

*These authors contributed equally to this study.

Corresponding author: L. Huang

E-mail: huangluqi@263.net

Genet. Mol. Res. 12 (4): 4827-4835 (2013)

Received March 23, 2013

Accepted August 15, 2013

Published October 22, 2013

DOI http://dx.doi.org/10.4238/2013.October.22.2

\begin{abstract}
Authenticating multi-species original raw materials in commercial formulations is difficult. Jin Yin Hua and Shan Yin Hua, both classified as raw honeysuckle materials in the Chinese Pharmacopoeia, are used in various medicines. Differentiating one variety from another is difficult based on chemical analysis. We developed molecular authentication of multi-species original honeysuckle in 3 brands of commercial tablets using allele-specific PCR. All 3 tablets contained both Jin Yin Hua and Shan Yin Hua. We also built a PCR-enzyme digestion method and enzymatic mutation detection in the PCR fragments of $p s b A$ trnH and trnL-trnF, and the restriction endonucleases HinfI and NlaIV, respectively. The PCR-enzyme digestion method produced the same result as the allele-specific PCR. Sequence and phylogenetic analyses show that the tablets YXC and YQJ contained Lonicera japonica and $L$. macranthoides as original raw materials, and LYG contained $L$. japonica, L. hypoglauca, and L. macranthoides.
\end{abstract}

Key words: SNP genotyping; Restriction endonuclease; Honeysuckle; Chloroplast sequences; Commercial tablets 


\section{INTRODUCTION}

Raw materials are primarily used in producing commercial tablets in Chinese material medicine. The compounds contained in original raw materials consisting of multiple species are different from those in materials composed of a single species. Differences in contents of tablets can be due to the use of different species and can result in different efficacy.

Honeysuckles are used in herbal medicines, and they possess biological and pharmaceutical properties such as anti-bacterial, anti-inflammatory, anti-viral, liver protectant, anti-angiogenic, and anti-nociceptive activities (Ku et al., 2009; Rahman and Kang, 2009; Ryu et al., 2010; Kang et al., 2010). Five species of honeysuckle, namely, Lonicera (L.) japonica, L. hypoglauca, L. macranthoides, L. confuse, and L. fulvotomentosa, are recorded in the Chinese Pharmacopoeia (Commission, 2010). Only L. japonica was indicated as Jin Yin Hua, and 4 other species represented Shan Yin Hua (Commission, 2010). Chlorogenic acid is used in evaluating the chemical quality of these 5 species, and both dipsacoside B and macranthoidin B are used only to evaluate L. hypoglauca, $L$. macranthoides, L. confuse, and L. fulvotomentosa (Chai et al., 2005; Shang et al., 2011). However, the similarities in morphological and biochemical characteristics prevent the application of chemical analysis in determining which species are used in producing a commercial tablet (Pu et al., 2002).

Chloroplast sequences are candidates for species recognition (Kress et al., 2005). Sun et al. (2011) published the sequences of $r b c L$, matK, psbA-trnH, and trnL-trnF of $L$. japonica and its closely related species. We collected and compared 1002 chloroplast sequences of Lonicera Linn. from the GenBank database and obtained 1 SNP (C/A) in trnLtrnF and 2 SNP (G/A, A/T) in psbA-trnH among L. japonica and its 18 closely related species, which include L. hypoglauca, L. macranthoides, L. confuse, and L. fulvotomentosa. Based on the SNP in $\operatorname{trnL}$-trnF, we designed an allele-specific polymerase chain reaction (PCR) method for the molecular authentication of L. japonica medicinal materials (Jiang et al., 2012).

In this study, we also developed a PCR-enzyme digestion method for the molecular authentication of L. japonica medicinal materials based on the SNP in $p s b A$-trnH. Three types of commercial tablets in Chinese material medicine that contain honeysuckle were initially submitted to molecular authentication using allele-specific PCR and a PCR-RFLP method. trnL-trnF sequences from the DNA of the commercial tablets were analyzed to determine which honeysuckle species were used in producing the tablets.

\section{MATERIAL AND METHODS}

\section{Sample collection}

Samples of honeysuckle including L. japonica, L. hypoglauca, L. macranthoides, L. confuse, and L. fulvotomentosa were collected from Guangxi, Henan, Shandong, Jiangsu, Beijing, and Anhui Provinces in China. All samples collected were identified by a taxonomist (Table 1). Three types of tablet samples, namely, Fu Fang Yu Xing Cao Pian, Lin Yang Gan Mao Pian, and Yin Qiao Jie Du Pian, were purchased from different pharmacies (Table 2). 


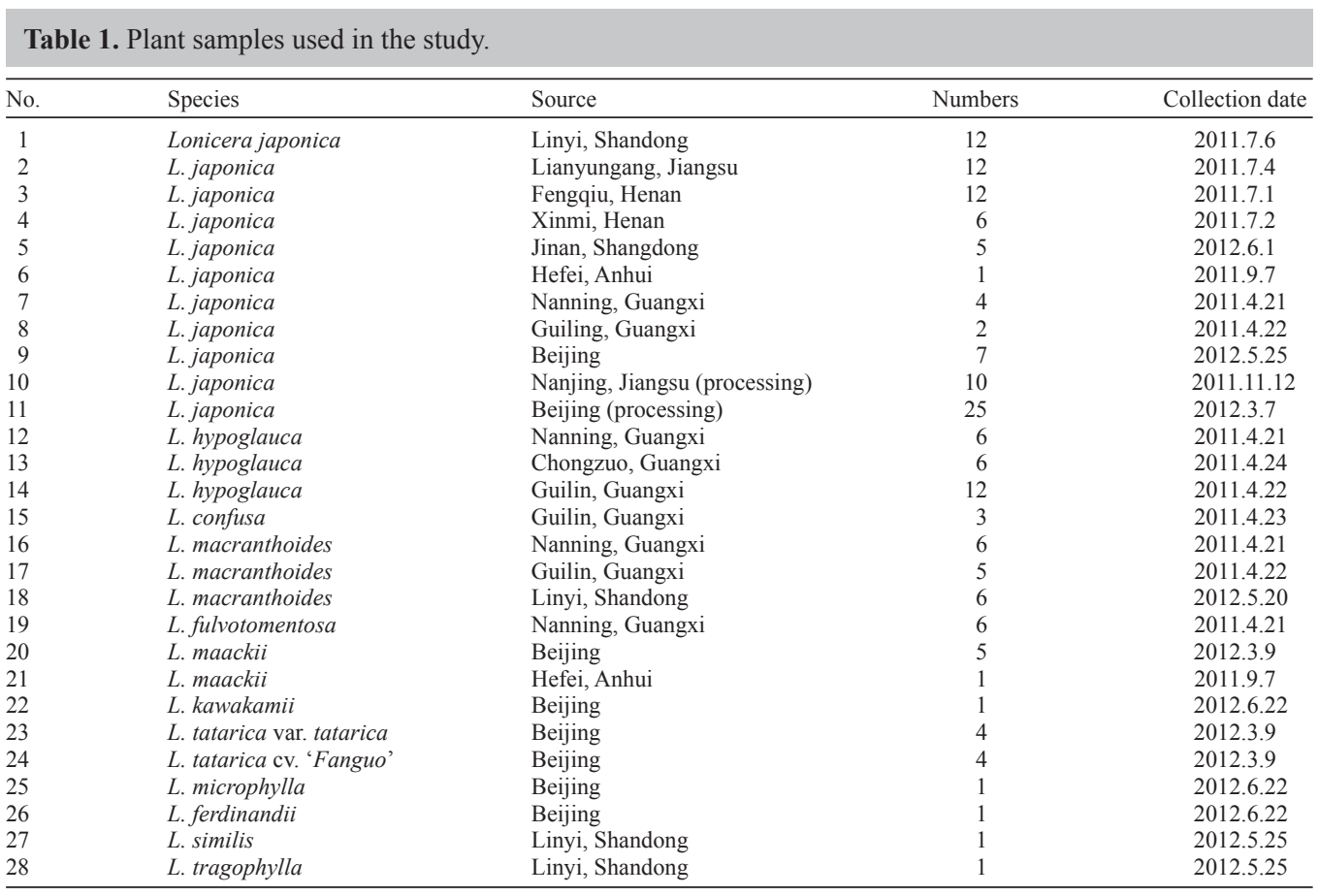

Table 2. Tablet samples used in the study.

\begin{tabular}{lll}
\hline No. & Tablet* & Raw materials \\
\hline YXC & Fu Fang Yu Xing Cao Pian & Honeysuckle, chameleon, weeping forsythia \\
LYG & Lin Yang Gan Mao Pian & Honeysuckle, platycodon root \\
YQJ & Yin Qiao Jie Du Pian & Honeysuckle, platycodon root \\
\hline
\end{tabular}

*Sequences in Supplementary material.

\section{Genomic DNA extraction and PCR amplification}

The genomic DNA was isolated from the sample (25 mg each) using the modified CTAB method ( $\mathrm{Li}$ et al., 2007). Total genomic DNA was used in the allele-specific PCR according to the protocol established by Jiang et al. (2012). PCR was performed by adding 2.5 $\mu \mathrm{L}$ 10X Ex Taq buffer, $2 \mu \mathrm{L} 10 \mathrm{mM}$ dNTPs, $0.5 \mathrm{U}$ Ex Taq (Takara, China), and $10 \mathrm{ng}$ DNA per reaction volume as well as 0.2 pmol of each forward and reverse primer. Total genomic DNA was also used in PCR to amplify trnL-trnF and psbA-trnH from L. japonica and its closely related species, respectively. The universal primers, sequences, and PCR conditions used in the amplification are presented in Table 3.

\section{Development of the PCR-enzyme digestion method}

Sequence assembly and consensus sequence generation were performed using BioEdit v. 7.1.3. The analysis of restriction endonuclease sites was performed using Primer Premier 
5.0 (Primer, Canada). The amplified PCR products of $\operatorname{trn} L-\operatorname{trn} F$ and $p s b A$-trnH were digested with NlaIV (recognition: $\mathrm{GGNN}^{\wedge} \mathrm{CC}$ ) and $\operatorname{HinfI}$ (recognition: $\mathrm{G}^{\wedge} \mathrm{ANTC}$ ), respectively.

\begin{tabular}{|c|c|c|c|}
\hline Primer & Name & Sequence $5^{\prime} \rightarrow 3^{\prime}$ & Reaction conditions \\
\hline \multirow{2}{*}{ Lonicera japonica primer } & $\mathrm{Lj}-1 \mathrm{~F}$ & GTTGACTGTCCTGTGTTGGT & $94^{\circ} \mathrm{C} 5 \mathrm{~min}$ \\
\hline & $\mathrm{Lj}-1 \mathrm{R}$ & TGAGAAATATAACGAATTTAG & $94^{\circ} \mathrm{C} 30 \mathrm{~s}, 54^{\circ} \mathrm{C} 30 \mathrm{~s}, 72^{\circ} \mathrm{C} 45 \mathrm{~s}, 35$ cycles, $72^{\circ} \mathrm{C} 7 \mathrm{~min}$ \\
\hline \multirow{2}{*}{ Non-Lonicera japonica primer } & $\mathrm{Lj}-2 \mathrm{~F}$ & TATCCTTTTTTTGTTAGCGGTTGA & $94^{\circ} \mathrm{C} 5 \mathrm{~min}$ \\
\hline & $\mathrm{Lj}-2 \mathrm{R}$ & CTATCCCGACCATTCCC & $94^{\circ} \mathrm{C} 30 \mathrm{~s}, 54^{\circ} \mathrm{C} 30 \mathrm{~s}, 72^{\circ} \mathrm{C} 45 \mathrm{~s}, 35$ cycles, $72^{\circ} \mathrm{C} 7 \mathrm{~min}$ \\
\hline \multirow[t]{2}{*}{ Universal primers 1} & $\operatorname{trnL}$ & CGAAATCGGTAGACGCTACG & $94^{\circ} \mathrm{C} 5 \mathrm{~min}$ \\
\hline & $\operatorname{trnF}$ & ATTTGAACTGGTGACACGAG & $94^{\circ} \mathrm{C} 30 \mathrm{~s}, 54^{\circ} \mathrm{C} 30 \mathrm{~s}, 72^{\circ} \mathrm{C} 45 \mathrm{~s}, 30$ cycles $^{*}, 72^{\circ} \mathrm{C} 7 \mathrm{~min}$ \\
\hline \multirow[t]{2}{*}{ Universal primers 2} & psbA & GTTATGCATGAACGTAATGCTC & $94^{\circ} \mathrm{C} 5 \mathrm{~min}$, \\
\hline & $\operatorname{trnH}$ & CGCGCATGGTGGATTCACAATCC & $94^{\circ} \mathrm{C} 30 \mathrm{~s}, 56^{\circ} \mathrm{C} 30 \mathrm{~s}, 72^{\circ} \mathrm{C} 30 \mathrm{~s}, 30$ cycles $^{*}, 72^{\circ} \mathrm{C} 7 \mathrm{~min}$ \\
\hline
\end{tabular}

*36 cycles for tablet samples.

\section{Cloning, sequencing, and sequence analysis}

The amplified PCR products of psbA-trnH were cloned into pMD19-T vector (Takara, Japan). Ten clones were randomly selected from each sample and directly sequenced at Beijing Genomics Institute, China, using a 3730XL sequencer (Applied Biosystems, USA). Sequence assembly and consensus sequence generation were performed using DNA STAR (Madison, WI, USA). The boundary of the psbA-trnH clones was determined according to the annotations of similar sequences in GenBank. To perform a phylogenetic study, the sequences of the DNA regions were aligned using ClustalW (Thompson et al., 1994), and the genetic distances were computed using MEGA 5.0 (Tamura et al., 2011) in accordance with the Kimura 2-parameter model.

\section{RESULTS AND DISCUSSION}

In recent years, several technologies such as morphology, chemical analysis (Xie et al., 2006), biological activity assay (Bai et al., 1997; Liu et al., 2006), and DNA molecular markers (Yip et al., 2007; Al-Qurainy et al., 2011; Guo et al., 2011) have been developed for authenticating raw herbal materials. With their characteristics of non-reliance on morphology, reproducibility, extreme reliability, and easy manipulation, DNA-based molecular tools are urgently needed in authenticating raw herbal materials. Authentication is essential for the standardization of Chinese medicines (Zhao et al., 2006). Authentication of raw materials is also required for commercial tablets in Chinese material medicine to maintain the quality of these tablets. However, few studies have focused on such molecular authentication. Although many studies have explored the identification of raw honeysuckle using DNA-based molecular tools, such as DNA barcoding (Sun et al., 2011), PCR-RFLP (Peng et al., 2010) and ITS sequencing (Hu et al., 2012), they have not focused on how to authenticate honeysuckle in tablets.

Coghlan et al. (2012) developed a high-throughput sequencing approach to authenticate the animal and plant composition of 15 traditional commercial Chinese medicine preparations, but this approach was too expensive and time-consuming. In this study, 3 types of commercial tablets containing honeysuckle were purchased from pharmacies and authenticated through the allele-specific PCR method. The results showed that all 3 types of commercial tablet contained L. japonica and its closely related species (Figure 1). 


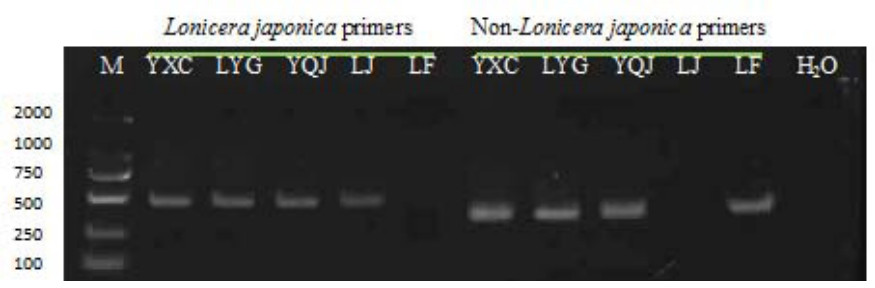

Figure 1. Allele-specific PCR using Lonicera japonica primers and non-Lonicera japonica primers. YXC, LYG, and YQJ $=3$ kinds of tablet samples, FFYXCP, LYGMP, and YQJDP. LJ = Lonicera japonica medical material; LF = L. fulvotomentosa medical material. Lane $M=$ DL2000 marker.

To verify the results, we developed an authentication method based on PCR-enzyme digestion. Based on the SNP (C/A) in trnL-trnF and SNP (G/A) in psbA-trnH between L.japonica and its closely related species, special restriction endonuclease sites, NlaIV and HinfI, were detected in chloroplast sequences of L. japonica. $\operatorname{trn} L-\operatorname{trn} F$ and $p s b A$-trnH fragments of 165 honeysuckle samples were amplified, and their PCR products were digested by NlaIV and HinfI. The results showed that PCR-NlaIV and HinfI digestion could be used in authenticating L. japonica and its closely related species (Figure 2). The tablets' PCR fragments amplified by $\operatorname{trnL}$-trnF and $p s b A$-trnH were digested by NlaIV and HinfI, respectively (Figure 3). The results were similar to those of the allele-specific PCR method.
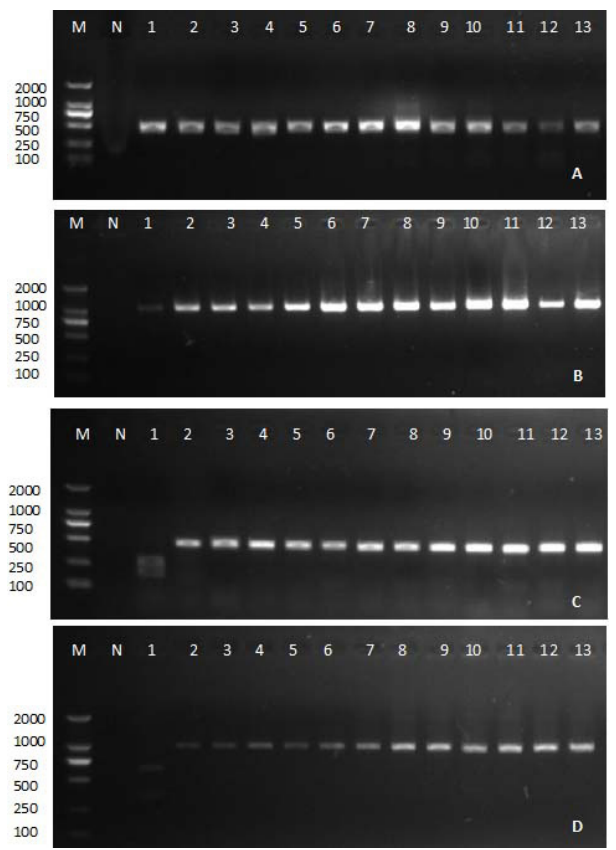

Figure 2. Authenticating honeysuckle samples using PCR-enzyme digestion. A. $p s b A$-trnH fragment; B. $\operatorname{trn} L-\operatorname{trn} F$ fragment; C. $p s b A$-trnH fragments digested by HinfI; D. $\operatorname{trnL}$-trnF fragments digested by NlaIV. Lane $M=\mathrm{DL}$ 2000 marker; lane $N=\mathrm{ddH}_{2} \mathrm{O}$ as negative control; lane $1=$ Lonicera japonica; lane $2=$ L. hypoglauca; lane $3=L$. confusa; lane $4=L$. macranthoides; lane $5=L$. maackii; lane $6=L$. fulvotomentosa; lane $7=L$. kawakamii; lane $8=$ L. tatarica var. tatarica; lane $9=$ L. tatarica cv. 'Fanguo'; lane $10=$ L. microphylla; lane $11=$ L. ferdinandii; lane $12=$ L. similis; lane $13=$ L. tragophylla. 


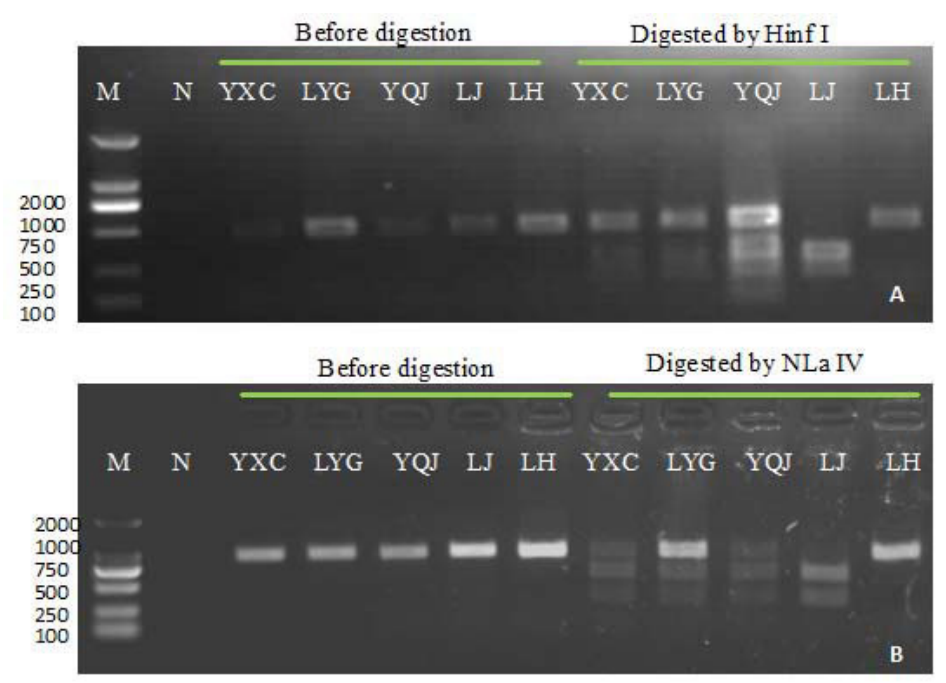

Figure 3. Endonuclease restriction analysis of 3 tablet samples. A. $p s b A$-trnH PCR productions digested by HinfI; B. $\operatorname{trn} L$-trnF PCR productions digested by NlaIV. Lane $M=\mathrm{DL} 2000$ marker; lane $N=\mathrm{ddH}_{2} \mathrm{O}$ as negative control; YXC, LYG, and YQJ = 3 kinds of tablet samples, FFYXCP, LYGMP, and YQJDP; LJ = Lonicera japonica; $\mathrm{LH}=$ L. hypoglauca.

However, aside from L. japonica, other species used in producing commercial tablets could still not be distinguished. Thus, we selected an accepted chloroplast sequence psbAtrnH and obtained a mixed PCR production from the DNA of the tablet. The mixed PCR production was cloned into PMD19-T vector and 10 single PCR clones were randomly selected and sequenced. Honeysuckle species in the tablets were distinguished using sequence and phylogenetic analyses.

Phylogenetic analysis showed that 2 clones in YXC, 4 clones in LYG, and 3 clones in YQJ were clustered with $L$. japonica, 8 clones in YXC with $L$. confuse, 4 clones in LYG with $L$. hypoglauca and $L$. macranthoides, and 7 clones in YQJ with $L$. macranthoides and $L$. confuse (Figure 4).

BLAST results also revealed 10 clones in YXC, 8 clones in LYG, and 10 clones in YQJ annotated with honeysuckle species. Further analysis showed that 2 clones in YXC and 3 clones in YQJ were annotated with $L$. japonica and the rest of the clones with L. macranthoides. Five clones in LYG were annotated with L. japonica, 1 clone with L. hypoglauca, and 2 clones with L. macranthoides (Table 4).

In conclusion, we suggest that the honeysuckle species $L$. japonica and $L$. macranthoides can be used in YXC and YQJ, and L. japonica, L. hypoglauca, and L. macranthoides can be used in LYG. Although these species have similar compositions, they differ slightly in terms of the content of active compounds (Pu et al., 2002; Ren et al., 2008). The content of chlorogenic acid is lower in L. japonica than in L. macranthoides and L. hypoglauca (Zhou and Tong, 2003; Chen et al., 2005). However, chlorogenic acid was the only compound used in evaluating the quality of the tablets, and the price of L. japonica was higher than that of other species. Thus, considering the suitable chlorogenic acid content and the production cost, mixed species of original honeysuckle were used in producing the tablets, thus making it difficult to distinguish the species used in these tablets by chemical analysis. 


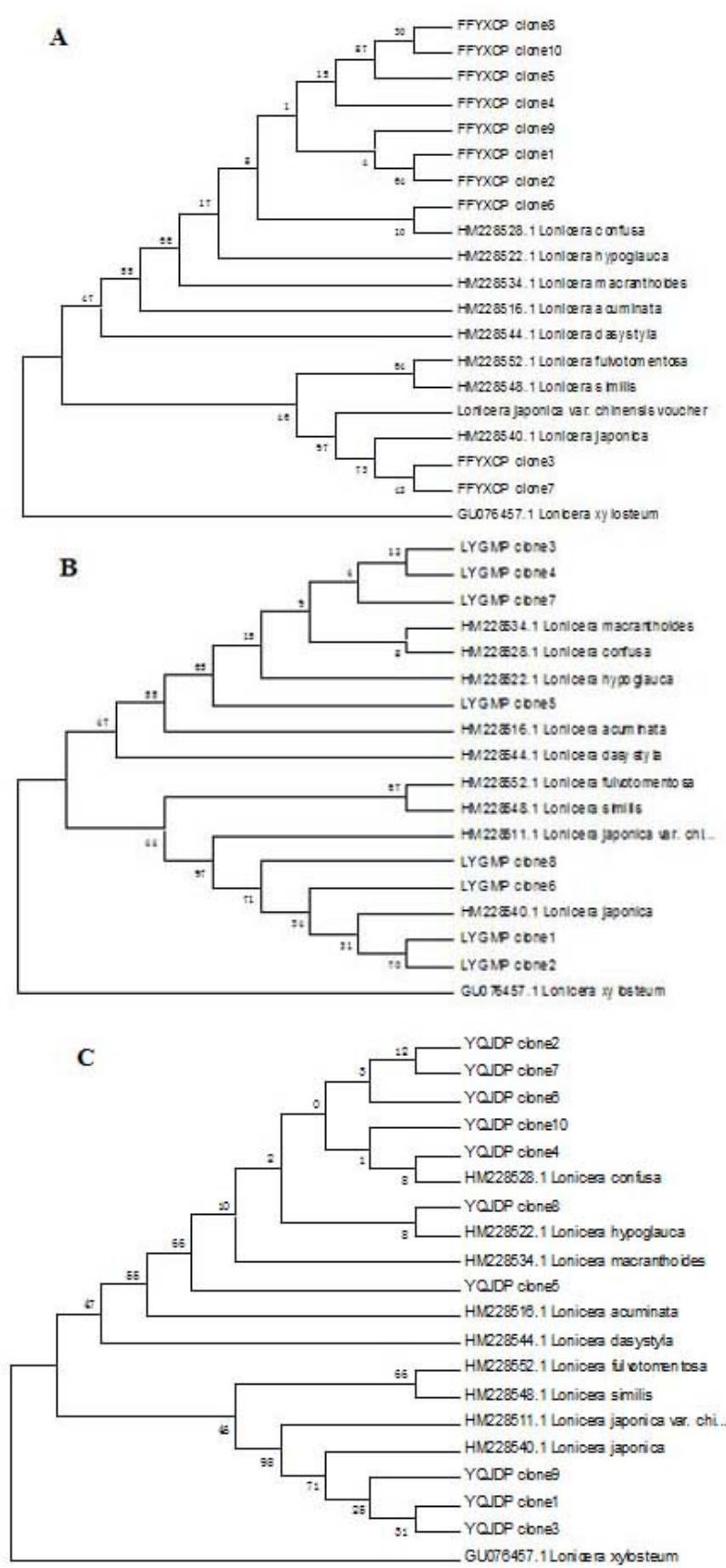

Figure 4. Phylogenetic analysis of psbA-trnH clones from 3 kinds of tablet samples and psbA-trnH sequences of Lonicera japonica, L. confuse, L. hypoglauca, L. macranthoides, L. acuminata, L. dasystyla, L. fulvotomentosa, L. similis, L. xylosteum from GenBank. A. Tablet Fu Fang Yu Xing Cao Pian (FFYXCP); B. tablet Ling Yang Gan Mao Pian (LYGMP); C. tablet Yin Qiao Jie Du Pian (YQJDP). 
Table 4. BLAST results of honeysuckle in three tablets.

\begin{tabular}{|c|c|c|c|c|c|c|c|}
\hline \multirow[t]{2}{*}{ No. } & \multirow[t]{2}{*}{ Tablet } & \multicolumn{3}{|c|}{ Total clones } & \multicolumn{3}{|c|}{ Honeysuckle clones } \\
\hline & & Total & Honeysuckle & Others & Total & L. japonica & Others \\
\hline YXC & Fu Fang Yu Xing Cao Pian & 10 & 10 & 0 & 10 & 2 & 8 \\
\hline LYG & Lin Yang Gan Mao Pian & 10 & 8 & 2 & 8 & 5 & 3 \\
\hline YQJ & Yin Qiao Jie Du Pian & 10 & 10 & 0 & 10 & 3 & 7 \\
\hline
\end{tabular}

We provide here a sequencing method to detect the species in complex prescriptions and to evaluate the quality of commercial tablets.

\section{Supplementary material}

\section{ACKNOWLEDGMENTS}

Research supported by the National Natural Science Foundation of China (\#81001605).

\section{REFERENCES}

Al-Qurainy F, Khan S, Tarroum M, Al-Hemaid FM, et al. (2011). Molecular authentication of the medicinal herb Ruta graveolens (Rutaceae) and an adulterant using nuclear and chloroplast DNA markers. Genet. Mol. Res. 10: 2806-2816.

Bai G, Fujiwara K, Tanimori H and Kitagawa T (1997). Development and application of a sandwich enzyme immunoassay for Glycyrrhizae Radix protein (GRP) using monoclonal antibodies. Biol. Pharm. Bull. 20: 1224-1228.

Chai XY, Li SL and Li P (2005). Quality evaluation of Flos lonicerae through a simultaneous determination of seven saponins by HPLC with ELSD. J. Chromatogr. A 1070: 43-48.

Chen J, Li SL, Li P, Song Y, et al. (2005). Qualitative and quantitative analysis of active flavonoids in Flos Lonicerae by capillary zone electrophoresis coupled with solid-phase extraction. J. Sep. Sci. 28: 365-372.

Coghlan ML, Haile J, Houston J, Murray DC, et al. (2012). Deep sequencing of plant and animal DNA contained within traditional Chinese medicines reveals legality issues and health safety concerns. PLoS Genet. 8: e1002657.

Commission CP (2010). The Pharmacopoeia of the People's Republic of China. Chinese Medicine Technology Press, Beijing, 205-206.

Guo X, Wang X, Su W, Zhang G, et al. (2011). DNA barcodes for discriminating the medicinal plant Scutellaria baicalensis (Lamiaceae) and its adulterants. Biol. Pharm. Bull. 34: 1198-1203.

Hu S, Dong G, Chen X, Huang L, et al. (2012). ITS sequence-based identification and utilization evaluation of "Nanjiang" (Lonicera similis Hemsl.), a local cultivar in Sichuan, China. Genet. Resour. Crop Evol. 59: 547.

Jiang C, Zhang Y, Chen M, Yuan Y, et al. (2012). Authenticate Lonicera japonica Thunb using bidirectional PCR amplification of specific alleles. ZhongGuo ZhongYao Za Zhi 37: 45-50.

Kang M, Jung I, Hur J, Kim SH, et al. (2010). The analgesic and anti-inflammatory effect of WIN-34B, a new herbal formula for osteoarthritis composed of Lonicera japonica Thunb and Anemarrhena asphodeloides BUNGE in vivo. J. Ethnopharmacol. 131: 485-496.

Kress WJ, Wurdack KJ, Zimmer EA, Weigt LA, et al. (2005). Use of DNA barcodes to identify flowering plants. Proc. Natl. Acad. Sci. U. S. A. 102: 8369-8374.

Ku SK, Seo BI, Park JH, Park GY, et al. (2009). Effect of Lonicerae Flos extracts on reflux esophagitis with antioxidant activity. World J. Gastroenterol. 15: 4799-4805.

Li JT, Yang J, Chen DC, Zhang XL, et al. (2007). An optimized mini-preparation method to obtain high-quality genomic DNA from mature leaves of sunflower. Genet. Mol. Res. 6: 1064-1071.

Liu N, Wen X, Liu J, Liang M, et al. (2006). Determination of ruscogenin in crude Chinese medicines and biological samples by immunoassay. Anal. Bioanal. Chem. 386: 1727-1733.

Peng X, Li W, Wang W and Bai G (2010). Identification of Lonicera japonica by PCR-RFLP and allele-specific diagnostic PCR based on sequences of internal transcribed spacer regions. Planta Med. 76: 497-499.

Pu Z, Xing J, Li P, Liu T, et al. (2002). Studies on the floral morphology of Flos Lonicerae. Zhong Yao Cai. 25: 854-859. Rahman A and Kang SC (2009). In vitro control of food-borne and food spoilage bacteria by essential oil and ethanol 
extracts of Lonicera japonica Thunb. Food Chem. 116: 670-675.

Ren MT, Chen J, Song Y, Sheng LS, et al. (2008). Identification and quantification of 32 bioactive compounds in Lonicera species by high performance liquid chromatography coupled with time-of-flight mass spectrometry. J. Pharm. Biomed. Anal. 48: 1351-1360.

Ryu KH, Rhee HI, Kim JH, Yoo H, et al. (2010). Anti-inflammatory and analgesic activities of SKLJI, a highly purified and injectable herbal extract of Lonicera japonica. Biosci. Biotechnol. Biochem. 74: 2022-2028.

Shang X, Pan H, Li M, Miao X, et al. (2011). Lonicera japonica Thunb.: ethnopharmacology, phytochemistry and pharmacology of an important traditional Chinese medicine. J. Ethnopharmacol. 138: 1-21.

Sun Z, Gao T, Yao H, Shi L, et al. (2011). Identification of Lonicera japonica and its related species using the DNA barcoding method. Planta Med. 77: 301-306.

Tamura K, Peterson D, Peterson N, Stecher G, et al. (2011). MEGA5: molecular evolutionary genetics analysis using maximum likelihood, evolutionary distance, and maximum parsimony methods. Mol. Biol. Evol. 28: 2731-2739.

Thompson JD, Higgins DG and Gibson TJ (1994). CLUSTAL W: improving the sensitivity of progressive multiple sequence alignment through sequence weighting, position-specific gap penalties and weight matrix choice. Nucleic Acids Res. 22: 4673-4680.

Xie P, Chen S, Liang YZ, Wang X, et al. (2006). Chromatographic fingerprint analysis - a rational approach for quality assessment of traditional Chinese herbal medicine. J. Chromatogr. A 1112: 171-180.

Yip PY, Chau CF, Mak CY and Kwan HS (2007). DNA methods for identification of Chinese medicinal materials. Chin. Med. 2: 9.

Zhao Z, Hu Y, Liang Z, Yuen JP, et al. (2006). Authentication is fundamental for standardization of Chinese medicines. Planta Med. 72: 865-874.

Zhou R and Tong Q (2003). Comparative study on content of chlorogenic acid in Lonicera japonica and L. macranthoides. Zhong Yao Cai. 26: 399-400. 